\title{
Comportamiento de seis clones de "cacao" (Theobroma cacao L.) en Guasaganda, provincia de Cotopaxi, Ecuador
}

\author{
Raúl Escobar
}

Coordinador del Programa de Ingeniería Agropecuaria, Guasaganda, Universidad Politécnica Salesiana. E-mail: decano_agro@ups.edu.ec

\section{Resumen}

En la actualidad, no existe una variedad nacional genéticamente pura del cacao, pues lo que se encuentra es una mezcla de híbridos naturales que se agrupan en una población conocida con el nombre de complejo "Nacional x Trinitario". Los tres grandes grupos de cacao que se reconocen a nivel internacional son: Criollos, Forasteros y Trinitarios, pero en el Ecuador existe una variedad Nacional que es diferente por ser nativa y proviene de los declives orientales de la Cordillera de los Andes en la hoya amazónica. La investigación se centró en monitorear el comportamiento de seis clones de cacao frente a las condiciones ambientales del sector de Guasaganda (cantón La Mana, provincia del Cotopaxi) y tendrá una duración de seis años, iniciando el 8 de marzo del 2004 y finalizando el 8 de marzo del 2010. Los resultados preliminares han sido el punto de partida para desarrollar proyectos socioeconómicos en el sector, ya que el experimento demostró que existe una precocidad de los clones EET-I03 y EET-544, porque se cosechan sus primeras mazorcas a los 12 meses de establecidos en el campo y la precocidad es un atributo asociado a la productividad, situación que favorece a los agricultores locales.

Palabras clave: Theobroma cacao L., cacao, clones, adaptación ambiental, precocidad, Ecuador.

\section{Introducción}

En la actualidad, se puede decir que prácticamente no existe una variedad nacional genéticamente pura del cacao, pues lo que se encuentra es una mezcla de híbridos naturales que se agrupan en una población conocida con el nombre de complejo "Nacional x Trinitario" (Loor y Amores 2003). A nivel botánico se reconocen tres grandes grupos de cacao que son: Criollos, Forasteros y Trinitarios, pero en el Ecuador existe una variedad Nacional que es diferente por ser nativa, ésta proviene de los declives orientales de la Cordillera de los Andes en la hoya amazónica y se conservó como exclusivo hasta 1890 , cuando se inició la introducción de material de origen Trinitario desde Venezuela (Wood et al. 1959).

A partir de la década de 1940, el Instituto Nacional Autónomo de Investigaciones Agropecuarias del
Ecuador (INIAP), a través del Programa Nacional de Cacao ha sumado esfuerzos para recolectar, estudiar y explotar el potencial del complejo "Nacional $x$ Trinitario" mediante la selección de clones con características de interés, puesto que éste se encuentra en un $95 \%$ de la superficie cacaotera del país. Así, una rigurosa selección fenotípica, basada en los caracteres de producción y resistencia a enfermedades, tuvo lugar en varias fincas cacaoteras de la zona central, y el producto fue un grupo de seis clones comerciales (EET-19, EET-48, EET-62, EET-95, EET-96, y EET-I03) que son distribuidos desde 1978.

La Estación Experimental Tropical Pichilingue del INIAP, dispone de clones de cacao cuyo comportamiento ha sido evaluado en algunas localidades de la Costa, donde tradicionalmente se encuentran las principales zonas cacaoteras del país. Sin embargo, el interés creciente por la explotación del cacao plantea 
inquietudes entre los productores acerca del comportamiento de dichos clones, especialmente en sectores con escasa tradición cacaotera y ambientes poco amigables para el cultivo por sus características edáficas y/o climáticas, como es el caso del sector de Guasaganda.

Dentro de este contexto, resaltan los sectores de La Maná y Pangua en la provincia de Cotopaxi, por ser reconocidos tradicionalmente como productores cacaoteros, hecho que lo demuestran los resultados de una serie de entrevistas aplicadas a una muestra de 110 productores cacaoteros en 42 recintos de las parroquias Guasaganda y Moraspungo (CAAP 2003). En este sentido, fue prioritario el estudio del comportamiento de varios clones de cacao en el área de Guasaganda, localizada en la estribación oriental de la Cordillera Andina, porque permitió: conocer y evaluar el comportamiento de seis clones de cacao como respuesta al entorno ambiental; seleccionar clones de cacao con mayor grado de adaptación de acuerdo a su desempeño agronómico, productivo y sanitario, considerando estándares establecidos para explotación comercial,y diseminar información sobre el manejo agronómico del cultivo en el sector a través de experiencias participativas en el campo y material divulgativo.
La investigación tendrá una duración de seis años, desde el 8 de marzo del 2004 hasta el 8 de marzo del 2010, tiempo necesario para monitorear el comportamiento de seis clones frente a las condiciones ambientales del sector de Guasaganda; por lo tanto, los resultados que se presentan son preliminares y se aspira que sean el punto de partida para desarrollar proyectos socioeconómicos como parte de la futura visión productiva del sector.

\section{Métodos de investigación}

I. Área del experimento: el estudio se ejecutó en la finca Sacha Wiwa del Sistema Educativo Intercultural Cotopaxi (SEIC), de la parroquia Guasaganda, cantón La Maná, provincia del Cotopaxi (Figura I). El área experimental está localizada a $512 \mathrm{msnm}$, presenta un clima subtropical húmedo, una temperatura promedio anual de $23,6^{\circ} \mathrm{C}$ y una precipitación media anual de $1.962,2 \mathrm{~mm}$. El suelo es de textura franco-arenosa y fertilidad relativamente baja que es característica del sector.

2. Equipo técnico: los investigadores que participan en el proyecto son: Ing. Freddy Amores P., Líder Nacional del Programa Nacional de Cacao y Café del

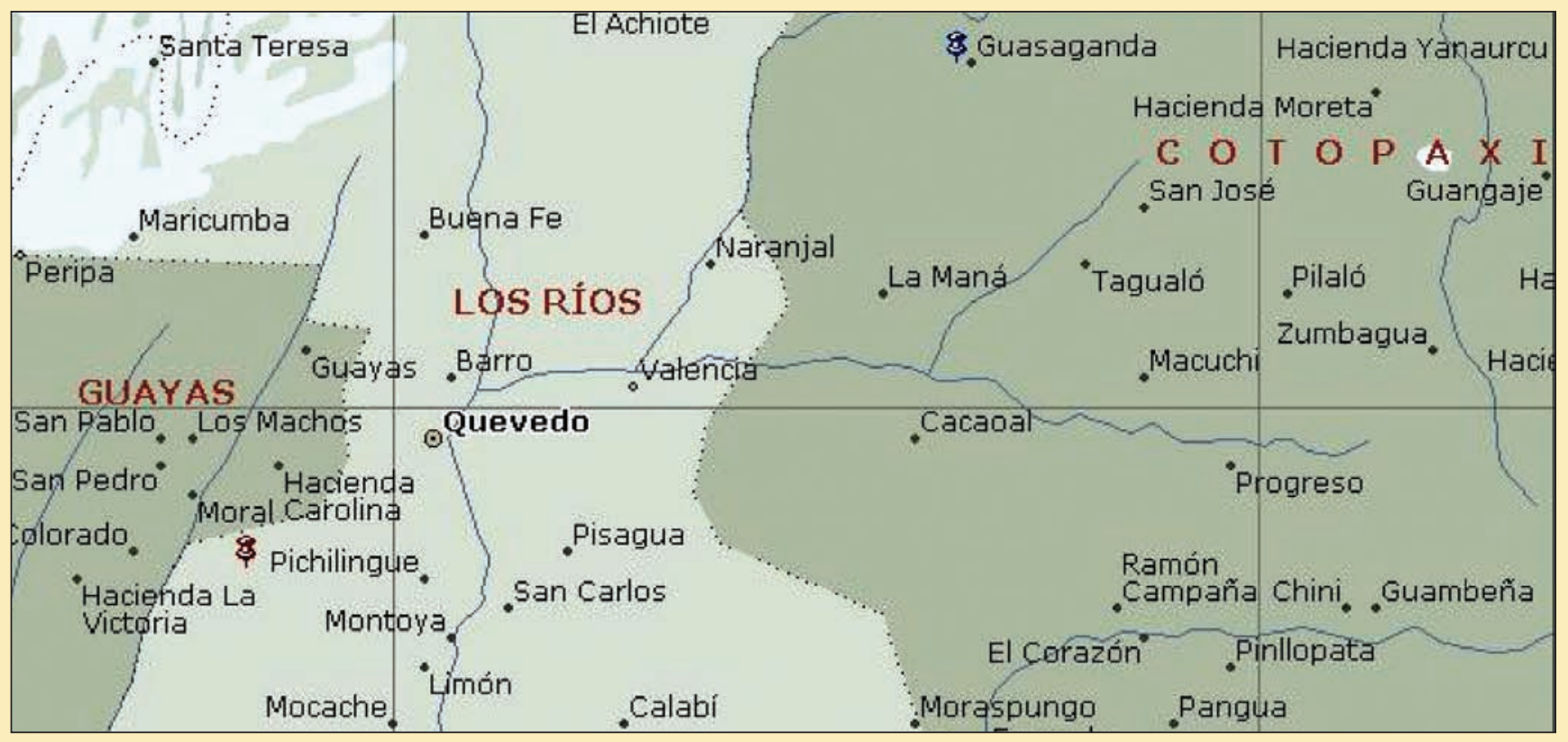

Figura I. Localización geográfica de la parroquia Guasaganda, cantón La Maná, provincia de Cotopaxi, Ecuador. 
INIAP; Ing. Alfonso Vasco M., investigador del Programa Nacional de Cacao y Café del INIAP; Lic. Francisco Rhón Dávila, Director Ejecutivo del Centro Andino de Acción Popular (CAAP), y Rommel Ramos R., Ing. Agrónomo egresado de la Universidad Técnica Estatal de Quevedo (UTEQ).

3. Análisis estadístico: los factores en estudio considerados fueron seis clones de cacao (EET-48, EET62, EET-96, EET-I03, EET-544 y CCN-5I) seleccionados a partir de sus características de producción y tolerancia a enfermedades (escoba de bruja y Monilia sp.). Los tratamientos (clones) están dispuestos en el campo mediante un diseño de bloques completos al azar con cuatro repeticiones e intercalados con plátano de la variedad Dominico Altón durante las primeras etapas de su desarrollo.

Las variables estuvieron representadas por los descriptores agronómicos recomendados por el Instituto Internacional de Recursos Fitogenéticos (IPGRI I98I) y los establecidos por el Programa de Cacao y Café del INIAP.

Los datos registrados para las variables seleccionadas fueron sometidos al análisis de varianza (ADV), prueba de rangos múltiples de Duncan $\mathrm{P} 0,05$ y análisis de correlaciones, porque estas pruebas estadísticas permitieron comparar el comportamiento anual de los seis clones en conjunto.

\section{Consideraciones finales}

Al finalizar los primeros cuatro años de evaluación del proyecto en el año 2008, se contó con un grupo de clones de cacao seleccionados por demostrar alta adaptación y se recomendó su cultivo en el sector de Guasaganda.

Los resultados preliminares del experimento demuestran que existe una precocidad de los clones EET-I03 y EET-544, porque mostraron sus primeras mazorcas a los 12 meses de establecidos en el campo. Por lo tanto, debe recordarse que la precocidad es un atributo asociado a la productividad y que se avizora un buen desempeño futuro de ambos clones de tipo Nacional (Tabla I).

El mayor número de mazorcas sanas lo presentaron los clones EET-I03, EET-544 y EET-96, sobresaliendo por el menor número de mazorcas enfermas con escoba de bruja el clon EET-544.

Al finalizar los diez años de experimentos con los seis clones de cacao, se espera contar con un grupo seleccionado que demuestre alta adaptación a factores ambientales y enfermedades, ya que esto permitiría recomendar su producción y explotación en el sector de Guasaganda. De este modo, el cultivo de cacao podría contribuir a que las poblaciones involucradas en esta actividad mejoren sus ingresos $y$, por lo tanto, su calidad de vida.

Tabla I. Resultados preliminares del experimento de identificación y genotipo de los seis clones de cacao con respecto al número de mazorcas sanas, número de mazorcas enfermas y escoba de bruja en la parroquia Guasaganda, cantón La Maná, provincia de Cotopaxi, Ecuador.

\begin{tabular}{lllccc}
\hline$N^{\circ}$ & Clon & Genotipo & $\begin{array}{c}\text { NMS/ } \\
\text { Tratamiento }\end{array}$ & $\begin{array}{c}\text { NME/ } \\
\text { Tratamiento }\end{array}$ & $\begin{array}{c}\text { NEB/ } \\
\text { Tratamiento }\end{array}$ \\
\hline I & EET-48 & Nacional & 8 & 3 & 14 \\
2 & EET-62 & Nacional x V.A. & 9 & 2 & 8 \\
3 & EET-96 & Nacional x V.A. & 90 & 4 & 10 \\
4 & EET-103 & Nacional x V.A. & 208 & 8 & 5 \\
5 & EET-544 & Nacional & 156 & 3 & 4 \\
6 & CCN-5I (testigo) & (ICS-95 IMC-67) $\times$ Canelo & 27 & 7 & 5 \\
\hline
\end{tabular}

CCN = Colección Castro Naranjal; EET = Estación Experimental Tropical Pichilingue; ICS = Imperial College Selection; IMC = lquitos Mixes Calabacillo; NEB = Número de escobas de bruja; NME = Número de mazorcas enfermas; NMS = Número de mazorcas sanas; Trat. = Tratamiento;V.A. = Venezolano Amarillo. 


\section{Literatura citada}

CAAP (Centro Andino de Acción Popular). 2003. Diagnóstico de la Situación Agrícola de los Cantones La Maná y Pangua, provincia del Cotopaxi. Quito, Ecuador. 56 pp.

IPGRI (International Plant Genetic Resources Institute). 1981. Genetic Resources of Cocoa. En: Workimg Group on Genetic Resources of Cocoa. Virginia, Estados Unidos. 25 pp.
Loor, R. y F. Amores. 2003. Explorando la variabilidad del cacao tipo Nacional para identificar clones elite. Revista Sabor Arriba 2(4): I 8- 19.

Wood, G.; A. R.; B. A. y DTA. 1959. El cacao en el Ecuador. En: Notes on Three Cocoa Diseases, Cocoa Growing in Venezuela, Colombia, and Ecuador. Cadbury Brothers Ltd. Bournville, Estados Unidos. Pp. 35-52.

El Programa Nacional de Cacao ha sumado esfuerzos para recolectar, estudiar y explotar el potencial de este recurso vegetal, especialmente mediante la selección de clones con características únicas.

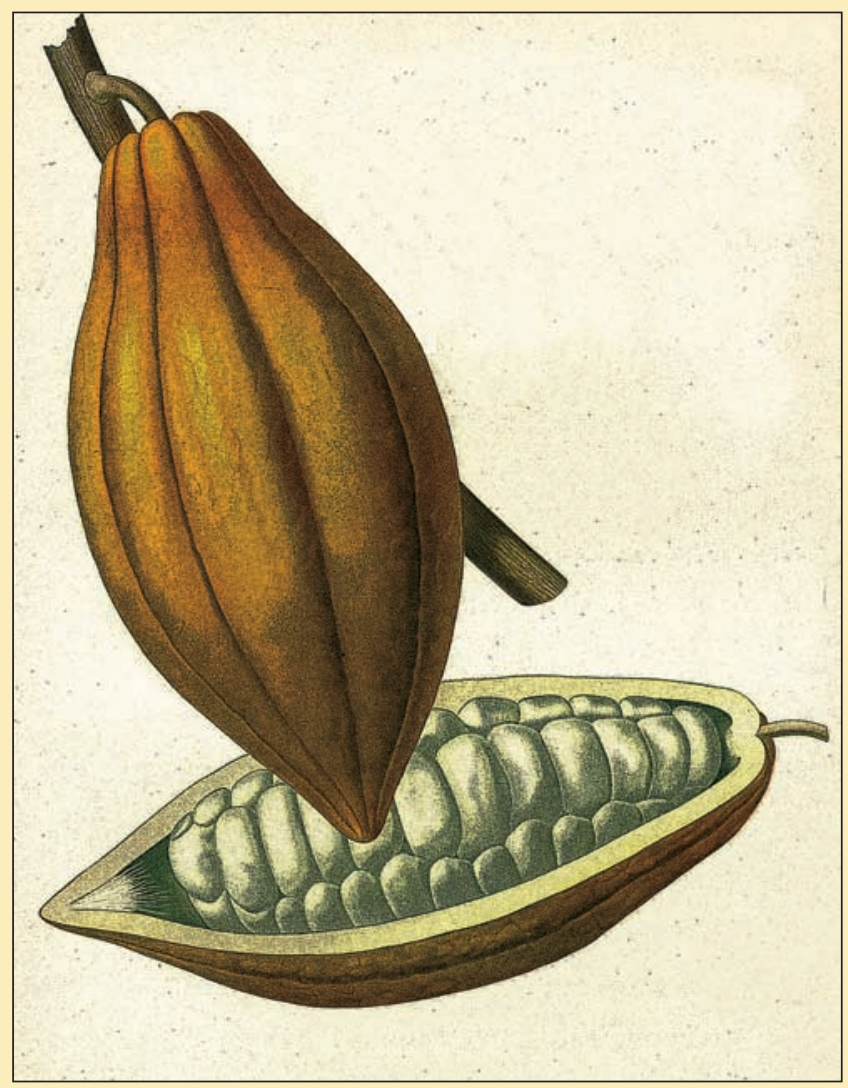

